\title{
Activation of retinoid $X$ receptor by bexarotene attenuates neuroinflammation via PPARY/SIRT6/FoxO3a pathway after subarachnoid hemorrhage in rats
}

Yuchun Zuo ${ }^{1,2}$, Lei Huang ${ }^{2,3}$, Budbazar Enkhjargal ${ }^{2}$, Weilin Xu², Ocak Umut², Zachary D. Travis ${ }^{6}$, Guangyu Zhang ${ }^{5}$, Jiping Tang ${ }^{2}$, Fei Liu ${ }^{1^{*}}$ and John H. Zhang ${ }^{2,3,4^{*}}$ (i)

\begin{abstract}
Background: Subarachnoid hemorrhage $(S A H)$ is a life-threatening subtype of stroke with high mortality and disabilities. Retinoid $X$ receptor (RXR) has been shown to be neuroprotective against ischemia/reperfusion injury. This study aimed to investigate the effects of the selective RXR agonist bexarotene on neuroinflammation in a rat model of SAH.

Methods: Two hundred male Sprague-Dawley rats were used. The endovascular perforation induced SAH. Bexarotene was administered intraperitoneally at $1 \mathrm{~h}$ after SAH induction. To investigate the underlying mechanism, the selective RXR antagonist UVI3003 and RXR siRNA or SIRT6 inhibitor OSS128167 was administered via intracerebroventricular $1 \mathrm{~h}$ before SAH induction. Post-SAH assessments including SAH grade, neurological score, brain water content, Western blot, and immunofluorescence were performed.

Results: The endogenous RXR and sirtuin 6 (SIRT6) protein levels were increased after SAH. Bexarotene treatment significantly reduced brain edema and improved the short-/long-term neurological deficit after SAH. Mechanistically, bexarotene increased the levels of PPARY and SIRT6; decreased the expression of phosphorylated FoxO3a ( $p$-FoxO3a), IL-6, IL-1 $\beta$, and TNF-a; and inhibited the microglia activation and neutrophils infiltration at $24 \mathrm{~h}$ after SAH. Either UVI3003, OSS128167, or RXR siRNA abolished the neuroprotective effects of bexarotene and its regulation on protein levels of PPARY/SIRT6/p-FoxO3a after SAH.
\end{abstract}

Conclusions: The activation of RXR by bexarotene attenuated neuroinflammation and improved neurological deficits after SAH. The anti-neuroinflammatory effect was at least partially through regulating PPARY/SIRT6/FoxO3a pathway. Bexarotene may be a promising therapeutic strategy in the management of SAH patients.

Keywords: Subarachnoid hemorrhage, Retinoid X receptor, Bexarotene, Sirtuin 6, Neuroinflammation, Brain edema

\section{Introduction}

Subarachnoid hemorrhage (SAH) is a devastating and life-threatening cerebrovascular disease with high mortality and disability. It accounts for $5 \%$ of all stroke subtypes, whereas caused $50 \%$ case fatality after aneurysm disruption [1]. Despite the availability of early imaging diagnosis and neurosurgical interventions (clipping) as

\footnotetext{
*Correspondence: doctorlf@126.com; johnzhang3910@yahoo.com 'Department of Neurosurgery, Third XiangYa Hospital, Central South University, No.138 Tongzipo Road, Changsha 410013, China

2Department of Physiology and Pharmacology, Loma Linda University, 11041 Campus St. Risley Hall, Loma Linda, CA 92354, USA

Full list of author information is available at the end of the article
}

well as platinum spirals (coiling), the high mortality and poor outcome of SAH result in a high socioeconomic burden [2]. Neuroinflammation is considered as one of the main pathological processes in the early brain injury (EBI) after SAH [3]. The brains residual microglial/astrocytes activation and peripheral immune cells infiltration release massive pro-inflammatory cytokines which would magnify the inflammatory responses and further aggravate the neurological deficit [4]. Herein, the therapeutic strategies targeting neuroinflammation would be practical to minimize EBI and improve neurologic outcomes after SAH.

(C) The Author(s). 2019 Open Access This article is distributed under the terms of the Creative Commons Attribution 4.0 International License (http://creativecommons.org/licenses/by/4.0/), which permits unrestricted use, distribution, and 
Retinoid X receptor (RXR) is a member of the NR2B nuclear receptor family. As a common binding partner of many other nuclear receptors, it mainly functions as a ligand-dependent transcription factor in regulating a plethora of physiological processes [5, 6]. RXR has been shown to bind with peroxisome proliferator-activated receptor gamma (PPAR $\gamma$ ) to form heterodimers [7]. PPAR $\gamma$ belongs to the nuclear hormone receptor superfamily and exerts an anti-inflammatory effect in many neurological diseases [8-12]. RXR agonists can activate RXR/PPAR $\gamma$ heterodimers to inhibit inflammatory response [7]. Recent studies demonstrated that PPARY activation induced the expression of sirtuin 6 (SIRT6) in a feed-forward manner $[13,14]$. Also, SIRT6-mediated suppression of forkhead box O3a (FoxO3a) phosphorylation protected cardiomyocytes against ischemia/reperfusion injury [15]. Thus, the regulation of the RXR/PPAR $/$ SIRT6/FoxO3a pathway may serve as a potential anti-neuroinflammation strategy in the setting of SAH.

Bexarotene (PubChem CID: 82146), a highly selective and blood-brain barrier permeable RXR agonist, is a member of the retinoic acid family [16]. It has been initially approved by the U.S. Food and Drug Administration (FDA) for treating cutaneous lymphoma with a favorable safety profile [17]. Recent studies suggested that bexarotene was neuroprotective against a variety of neurological diseases, such as Alzheimer's disease, traumatic brain injury, and ischemic stroke $[16,18,19]$. However, the neuroprotective effect of bexarotene and the underlying mechanisms have not been investigated in SAH.

In the present study, we hypothesized that bexarotene could attenuate neuroinflammation and improve neurological outcomes in a rat model of SAH. Such neuroprotective effects were mediated through PPARy/SIRT6/ FoxO3a signaling pathway.

\section{Materials and methods}

\section{Animals and the SAH models}

Adult male Sprague-Dawley rats $(n=200 ; 280-320$ g) were housed in a room with constant temperature $\left(25^{\circ} \mathrm{C}\right)$ and humidity control, under a 12-h light/dark cycle and free access to food and water. All the experimental procedures were approved by the Institutional Animal Care and Use Committee at Loma Linda University and in accordance with the National Institutes of Health Guide for the Care and Use of Laboratory Animals of the National Institutes of Health.

Experimental SAH model was induced in rats using a modified endovascular perforation method as previously described [20,21]. Briefly, rats were anesthetized by $5 \%$ isoflurane. After intubation, the rats were ventilated with $3 \%$ isoflurane in mixed gases $(65 \%$ medical air with $35 \%$ oxygen gas) during surgery. Rats were operated in the supine position, and the midline incision was made in the neck. After carefully exposing the left carotid artery, a 3-cm-long sharpened monofilament nylon suture (4-0) was inserted into the external carotid artery, advanced via the internal carotid to the bifurcation of the anterior and middle cerebral arteries where the resistance was felt, and then further advanced slightly $(3 \mathrm{~mm})$ to induce a hemorrhage. Afterward, the nylon suture was withdrawn immediately. The sham-operated animals underwent the same surgical procedure without arterial perforation. At the end of surgery, the incision was closed, and the tracheal tube was extubated. The rats were housed individually in heated cages after recovery from anesthesia.

\section{Experimental design}

Animals were randomly assigned to four separate experiments as following described. The information of experimental groups was blinded to the researchers who performed the surgeries, neurobehavioral assessments, Western blot, immunofluorescence staining, and data analysis. The experimental designs are schematically shown in Fig. 1.

\section{Experiment 1}

The time course and cellular localization of RXR and SIRT6 in the left hemisphere were accessed by Western blot and double immunofluorescence. Total of 36 rats were divided randomly and assigned into 6 groups with $n=6$ / group: sham and SAH 3, 6, 12, 24, and $72 \mathrm{~h}$ ( $n=6$ /group). The temporal pattern of RXR and SIRT6 expression was detected by Western blot. The additional four rats in sham and SAH $24 \mathrm{~h}$ group ( $n=2$ /group) were used for double immunofluorescence staining to show the co-localization of RXR with the neurons, astrocytes, and microglia.

\section{Experiment 2}

To assess the neuroprotective effects of bexarotene (Bexa), a total of 30 rats were randomized into 5 groups with $n=6$ /group: sham, $\mathrm{SAH}+$ vehicle, $\mathrm{SAH}+\mathrm{Bexa}$ $(5 \mathrm{mg} / \mathrm{kg}), \mathrm{SAH}+$ Bexa $(15 \mathrm{mg} / \mathrm{kg})$, and $\mathrm{SAH}+$ Bexa $(45 \mathrm{mg} / \mathrm{kg})$. Bexarotene was administered at $1 \mathrm{~h}$ after $\mathrm{SAH}$ by intraperitoneal (i.p) injection. Neurological functions and brain water content were measured at $24 \mathrm{~h}$ after SAH. Based on neurological tests and brain water content results, bexarotene at a dose of $15 \mathrm{mg} / \mathrm{kg}$ was the most effective. Thus this best dosage was chosen for the following long-term neurobehavior and mechanism experiments.

To explore the effects of bexarotene on microglia/ macrophage activation and neutrophil infiltration at $24 \mathrm{~h}$ after $\mathrm{SAH}$, another set of 30 rats were randomly divided into 3 groups with $n=10$ /group: sham, SAH + vehicle, and $\mathrm{SAH}+$ Bexa $(15 \mathrm{mg} / \mathrm{kg})$. Western blot $(n=6 /$ group $)$ and immunofluorescence staining $(n=4 /$ group $)$ were performed to detect the ionized calcium binding adaptor 


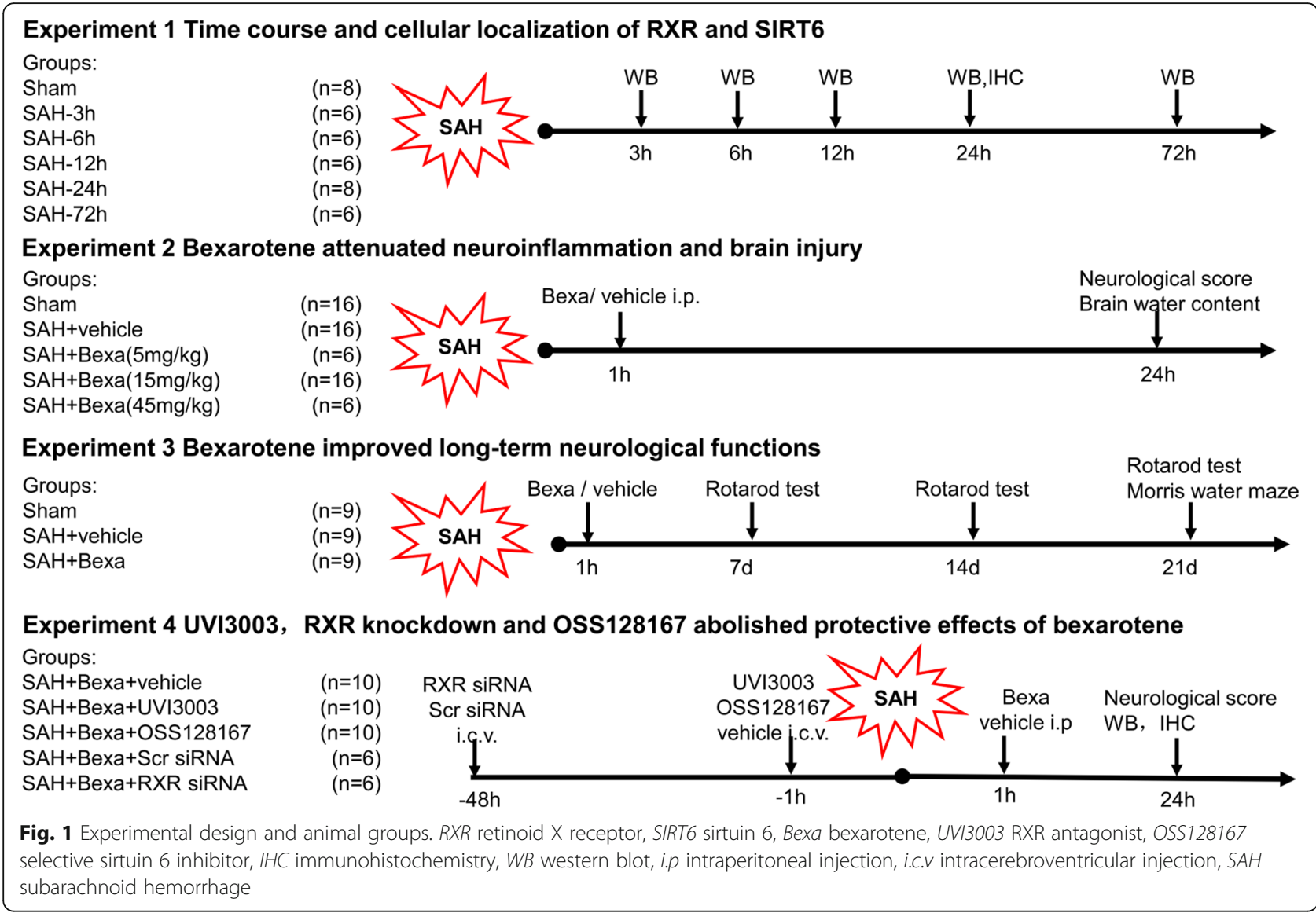

molecule 1 (Iba-1)-positive microglia and myeloperoxidase (MPO)-positive neutrophils at $24 \mathrm{~h}$ after SAH. Brain samples of these three groups were shared with experiment 4 .

\section{Experiment 3}

To evaluate the effects of bexarotene on long-term neurobehavioral recovery, 27 rats were randomly divided into 3 groups with $n=9$ /group: sham, $\mathrm{SAH}+$ vehicle, and $\mathrm{SAH}$ + Bexa $(15 \mathrm{mg} / \mathrm{kg})$. The rotarod tests were performed on days 7, 14, and 21 after SAH and Morris water maze test were performed on days 21-25 after SAH.

\section{Experiment 4}

To explore the potential mechanism of RXR/PPAR $\gamma /$ SIRT6/FoxO3a underlying Bexa mediated anti-neuroinflammation effects after SAH, the selective RXR inhibitor UVI3003 or selective SIRT6 antagonist OSS128167 was administration by intracerebroventricular (i.c.v) injection $1 \mathrm{~h}$ before SAH. To further prove the pathway, the RXR siRNA was administration by i.c.v injection $48 \mathrm{~h}$ before SAH. In addition to the shared brain samples of sham, $\mathrm{SAH}+$ vehicle, $\mathrm{SAH}+$ Bexa groups from experiment 2, additional 42 rats were assigned into 5 groups: $\mathrm{SAH}+\mathrm{Bexa}+$ vehicle $(n$ $=10$ /group), SAH + Bexa + UVI3003 ( $n=10$ /group), SAH + Bexa + OSS128167 ( $n=10$ /group $),$ SAH + Bexa +
Sramble siRNA ( $n=6$ /group), and SAH + Bexa + RXR siRNA ( $n=6$ /group). Neurological tests and Western blot and immunofluorescence were performed at $24 \mathrm{~h}$ after SAH.

\section{Drug administration}

Bexarotene (Selleck Chemicals, Houston, USA) was diluted in $10 \%$ dimethyl sulfoxide (DMSO). Administered $1 \mathrm{~h}$ after SAH by i.p injection, three different doses $(5 \mathrm{mg} / \mathrm{kg}$, $15 \mathrm{mg} / \mathrm{kg}$, and $45 \mathrm{mg} / \mathrm{kg}$ ) were evaluated. The SAH + vehicle group received an equal volume of $10 \%$ DMSO. The RXR inhibitor UVI3003 (75 $\mu \mathrm{g} / \mathrm{rat}$, Sigma-Aldrich, St. Louis, MO) or selective SIRT6 antagonist OSS128167 $(100 \mu \mathrm{g} / \mathrm{rat}$, Selleck Chemicals, Houston, TX) dissolved in $10 \%$ DMSO was injected by i.c.v route at $1 \mathrm{~h}$ before SAH. The same volume of $10 \%$ DMSO was injected via i.c.v in $\mathrm{SAH}+$ Bexa + vehicle group.

\section{Intracerebroventricular injection}

An intracerebroventricular injection was performed as described previously $[22,23]$. Briefly, rats were placed in a stereotaxic apparatus under anesthesia with $3 \%$ isoflurane in mixed gases ( $70 \%$ medical air/30\% oxygen). A cranial burr hole was drilled at the following coordinates relative to bregma: $1.5 \mathrm{~mm}$ posterior, $1.0 \mathrm{~mm}$ lateral. 
The 10- $\mu \mathrm{L}$ Hamilton syringe (Microliter701; Hamilton Company, Reno, NV, USA) was inserted at a depth of $3.3 \mathrm{~mm}$ into the right lateral ventricle through the burr hole as mentioned above. The needle was inserted through the burr hole. The rate of injection controlled at $1 \mu \mathrm{L} / \mathrm{min}$ with an infusion pump (Stoelting, Harvard Apparatus, Holliston, MA, USA). For RXR knockdown in vivo, rat RXR siRNA (Santa Cruz Technology, USA) or Scramble siRNA was prepared in RNase free suspension buffer at a concentration of $100 \mathrm{pmol} / \mathrm{uL}$ and then infused into the right lateral ventricle at $48 \mathrm{~h}$ before SAH induction. The needle was kept in place for an additional $5 \mathrm{~min}$ after the end of infusion and then slowly retracted throughout $5 \mathrm{~min}$. The burr hole was sealed with bone wax, and the rats were allowed to recover after sutures.

\section{SAH grading score}

The severity of SAH was evaluated according to a grading system as previously described [24]. Briefly, the basal cistern was divided into six segments and scoring based on the number of subarachnoid blood clots respectively by the following criteria: 0 , no subarachnoid blood; 1, minimal subarachnoid clots; 2, moderate subarachnoid clots with recognizable arteries; and 3, blood clots covering all arteries. The total score was obtained by adding the scores of all six segments. Rats with mild SAH (SAH grade score less than 8) at $24 \mathrm{~h}$ were excluded from this study. The grading was performed by researchers blinded to the experiment group information.

\section{Assessment of short-term neurological function}

Short-term neurological function was assessed using the modified Garcia scoring system and the beam balance test at $24 \mathrm{~h}$ after $\mathrm{SAH}$, as previously described [25]. The Garcia score was composed of six test subscores, including spontaneous activity, spontaneous movement of the four limbs, body proprioception, whisker proprioception, forepaw outstretching, and climbing. The total score ranging from 3 to 18 was obtained by adding the six test subscores [26]. Beam balance tests evaluated the ability of animals to walk on a narrow cylindrical wooden beam for $60 \mathrm{~s}$. The score was evaluated as follows: 0 , no walking and falling; 1 , no walking, but remains on the beam; 2 , walking but falling; 3, walking less than $20 \mathrm{~cm}$; 4, walking beyond $20 \mathrm{~cm}$. These neurological tests with high scores indicated better neurological function.

\section{Assessment of long-term neurological function}

Long-term neurobehavioral effects were assessed using the rotarod test and Morris water maze test as previously reported [27]. The rotarod test was performed to measure the balance and coordination abilities. Briefly, the rats were placed on the rotarod at the starting rate of $5 \mathrm{rpm}$ and $10 \mathrm{rpm}$ with an acceleration of 2 revolutions per $5 \mathrm{~s}$. The duration of rats on the rotarod were recorded and used for statistical analysis. The Morris water maze was used to evaluate the abilities of spatial learning and memory on days $21-25$ after SAH. Briefly, the rats were placed in different start locations using a semi-random method to find a visible platform above the water level in $60 \mathrm{~s}$. On the last day, probe trial test were performed with the platform removed. Swim trace and distance, escape latency, and probe quadrant duration were recorded by a computerized tracking system (Noldus Ethovision; Noldus, Tacoma, WA, USA).

\section{Brain water content}

Brain water content was evaluated by a wet/dry method, as described previously [28]. The brains were separated into four parts (left hemisphere, right hemisphere, cerebellum, and brain stem), and weighed immediately (wet weight), then weighed again after dried at $105{ }^{\circ} \mathrm{C}$ for $72 \mathrm{~h}$ (dry weight). The percentage of brain water content was calculated by [(wet weight - dry weight)/wet weight $] \times 100 \%$.

\section{Liquid chromatography-mass spectrometry}

The liquid chromatography-mass spectrometry (LC-MS/ MS) detection was performed as previously described [29]. The brain samples were prepared to follow the reported method [30]. Briefly, $200 \mathrm{mg}$ of brain tissue was homogenized in $2 \mathrm{~mL}$ of acetonitrile (Sigma-Aldrich, USA), and then centrifuged at $14,000 \mathrm{~g}$ for $30 \mathrm{~min}$ at $4^{\circ}$ $\mathrm{C}$. The supernatant was collected and dried under negative pressure (below $2.0 \mathrm{kPa}$ ) for $7 \mathrm{~h}$ at $4{ }^{\circ} \mathrm{C}$. The residue was reconstituted with $1000 \mu \mathrm{L} 50 \%$ acetonitrile and centrifuged at $14,000 \times g$ for $10 \mathrm{~min}$ at $4{ }^{\circ} \mathrm{C}$. Then, $20 \mu \mathrm{L}$ of the supernatant was injected into the LC-MS/MS system. The MS spectra were collected under the positive reflector mode from $\mathrm{m} / z$ 100-1000. MS/MS spectra were acquired using collision energy of $30 \mathrm{kV}$ with the metastable suppressor on. The LC-MS/MS data was visualized and analyzed by MassHunter Software Version B.08.00 (Agilent Technologies, CA, USA).

\section{Western blot analysis}

Western blotting was performed as described previously [31]. Briefly, the samples were extracted in RIPA buffer (Santa Cruz Biotechnology, CA, USA) and centrifuged with $14,000 \times g$ at $4{ }^{\circ} \mathrm{C}$ for $30 \mathrm{~min}$. The supernatant was collected and followed by a protein concentration measurement using detergent compatible assay (DC protein assay, Bio-Rad Laboratories, CA, USA). Equal amounts of protein samples were separated by SDS-PAGE gel and transferred to nitrocellulose membrane. Afterward, the membranes were blocked and incubated overnight at $4{ }^{\circ} \mathrm{C}$ with the following primary antibodies: anti-RXR 
(1:300; Santa Cruz Biotechnology, Santa Cruz, CA, USA), anti-PPARY (1:1000; Abcam, Cambridge, MA, USA), anti-SIRT6 (1:1000; Abcam, Cambridge, MA, USA), anti-phosphorylated FoxO3a (p-FoxO3a, 1:1000 Abcam, Cambridge, MA, USA), anti-FoxO3a (1:1000, Cell Signaling, Danvers, MA, USA), anti-Iba-1 (1:1000, Abcam, Cambridge, MA, USA), anti-MPO (1:500, Abcam, Cambridge, MA, USA), anti-IL-1 $\beta$ (1:1000, Abcam, Cambridge, MA, USA), anti-IL-6 (1:1000, Abcam, Cambridge, MA, USA), anti-TNF- $\alpha$ (1:1000, Abcam, Cambridge, MA, USA), and anti- $\beta$-actin (1:3000, Santa Cruz, Dallas, TX, USA). Appropriate secondary antibodies (1:3000, Santa Cruz, Dallas, TX, USA) were incubated at room temperature for $2 \mathrm{~h}$. The specific bands were visualized by an ECL reagent (Amersham Biosciences, Pittsburgh, PA). The relative densities of the immunoblot bands were analyzed using ImageJ software (Image J 1.4, NIH, USA).

\section{Immunoprecipitation (IP) detection}

The left hemisphere was lysed and extraction followed by centrifugation. The primary antibodies $(5 \mu \mathrm{g}$, anti-RXR, Santa Cruz Biotechnology, USA) were pre-incubated at room temperature mixed with $30 \mu \mathrm{L}$ agarose-G for $5 \mathrm{~h}$. After rinsed with GLB + buffer for five times, the brain lysate was added and incubated with agarose-G combined with primary antibody at $4{ }^{\circ} \mathrm{C}$ for $24 \mathrm{~h}$. After that, the mixed proteins were washed with pre-cold GLB + buffer three times, followed by eluted with $1 \times$ loading buffer in boiling water for $8 \mathrm{~min}$, and collected the supernatant after centrifuged at 12,000 $\mathrm{rpm}$ for $2 \mathrm{~min}$. The supernatant was loaded to SDS-PAGE.

\section{Immunofluorescence}

Rats were under deep anesthesia and transcardially perfused with pre-cold PBS and 10\% formalin. The brains were post-fixed in $10 \%$ formalin at $4{ }^{\circ} \mathrm{C}$ for $24 \mathrm{~h}$ followed by dehydrated in $30 \%$ sucrose for another $72 \mathrm{~h}$. Brain samples were frozen at $-80{ }^{\circ} \mathrm{C}$ after embedding in OCT and cut into $10-\mu \mathrm{m}$-thick coronal sections using a cryostat (CM3050S; Leica Microsystems, Bannockburn, III, Germany). To perform the immunofluorescence staining, the slices were rinsed and blocked with 5\% donkey serum at room temperature for $1 \mathrm{~h}$, then incubated overnight at $4{ }^{\circ} \mathrm{C}$ with the following primary antibodies: goat anti-Iba-1 (1:200, Abcam, Cambridge, MA, USA), goat anti-GFAP (1:200, Abcam, Cambridge, MA, USA), goat anti-NeuN (1:200, Abcam, Cambridge, MA, USA), mouse anti-RXR(1:100; Santa Cruz, Dallas, TX, USA), rabbit anti-SIRT6 (1:200, Abcam, Cambridge, MA, USA), rabbit anti-IL-1 $\beta$ (1:100, Abcam, Cambridge, MA, USA), and mouse anti-MPO (1:100, Santa Cruz, Dallas, TX, USA). After that, slides were incubated with the corresponding secondary antibodies (1:200, Jackson
Immunoresearch, West Grove, PA, USA). The sections were visualized and photographed under a fluorescence microscope (Leica Microsystems, Germany). Microphotographs were analyzed with LASX software. The numbers of Iba-1-positive cells, MPO-positive cells, and IL-1 $\beta$ positive cells were identified and counted in three different fields of the left basal cortex from five random coronal sections per rat, and data were expressed as cells/field.

\section{Quantification and statistical analysis}

The sample size was calculated based on the literature reviewing and our previous experimental SAH studies as well as the preliminary data collected for the treatment efficacy of Bexarotene against stroke. Using sample size calculator (SigmaPlot) with a power of 0.8 , and an alpha of 0.05 on a two-sided test, the animal numbers $n=6 /$ group was deemed sufficient to the experiments. All data were expressed as mean and standard deviation (means \pm SD) and plotted using Graph Pad Prism 7 (Graph Pad Software, San Diego, CA). SPSS 16.0 software (SPSS Inc., Chicago, Illinois, USA) was used for statistical analysis. One-way analysis of variance (ANOVA) followed by Tukey's post-hoc test was used for multiple comparisons among groups. Two-way ANOVA was applied to analyze long-term neurobehavioral results. $P<0.05$ was defined as statistical significance.

\section{Results}

\section{Animal mortality and SAH severity}

A total of 200 rats were used, in which 33 rats underwent a sham operation, and 163 rats underwent SAH induction. Out of 163 SAH rats, 4 rats were excluded because their SAH grading was less than 8 . The total mortality of SAH rats in the study was $16.56 \%(27 / 163)$. None of the rats died in the sham-operated group. The SAH mortality rate was not significantly different among the experimental groups. The average SAH grades among all the SAH groups were not statistically different. Blood clots were mainly distributed around the Circle of Willis and ventral brain stem after SAH induction, whereas no blood clot was observed in the sham operation (Additional file 1: Figure S1).

\section{Temporal patterns and cellular expression of endogenous brain RXR and SIRT6 after SAH}

Western blotting was performed to detect the protein levels of RXR and SIRT6 in the left hemisphere among groups of sham, 3, 6, 12, 24, and $72 \mathrm{~h}$ after SAH. The results showed that the expression of RXR and SIRT6 started increasing at $3 \mathrm{~h}$ after SAH and peaked at $24 \mathrm{~h}$ $(P<0.05$; Fig. 1a, b). The RXR and SIRT6 protein levels 
at $24 \mathrm{~h}$ post-SAH were nearly three times higher than those in the sham group. Double immunofluorescence staining of the RXR with the neurons marker NeuN, astrocytes marker glial fibrillary acidic protein (GFAP), or microglia marker Iba-1 showed that RXR expressed on the three types of cells within cortices in sham and SAH rats at $24 \mathrm{~h}$ after injury (Fig. 2c, d).

\section{Bexarotene treatment improved short-term}

\section{neurobehavioral deficits and reduced brain edema at}

\section{$24 \mathrm{~h}$ after $\mathrm{SAH}$}

The neurobehavioral outcomes and brain water content were evaluated at $24 \mathrm{~h}$ after SAH. Rats in the $\mathrm{SAH}+$ vehicle groups performed significantly worse than shams in the modified Garcia test and beam balance test $(P<0.01$; Fig. 3a, b). The administration of bexarotene $(15 \mathrm{mg} / \mathrm{kg}$ and $45 \mathrm{mg} / \mathrm{kg})$ significantly improved the neurological scores at $24 \mathrm{~h}$ after SAH. The brain water content in the left and right hemisphere was significantly increased in the SAH groups compared to sham at 24 after SAH $(P<0.05$, Fig. $3 c)$, which was significantly reduced by the administration of Bexa at a dose of $15 \mathrm{mg} / \mathrm{kg}(P<0.05$, Fig. 3c). The brain water contents in the cerebellum and brain stem were not significant differences between the sham and SAH groups. Bexarotene at $15 \mathrm{mg} / \mathrm{kg}$ was the most effective dosage, which was chosen for the following long-term and mechanistic studies.

\section{Bexarotene treatment reduced microglia/macrophage activations and neutrophil infiltration at $24 \mathrm{~h}$ after SAH}

The immunofluorescence staining of Iba-1- and MPO-positive cells and Western blot of Iba-1 and MPO protein levels were performed to access the microglia/ macrophage activation and neutrophil infiltration in sham, SAH + vehicle, and SAH + Bexa groups at $24 \mathrm{~h}$ after SAH. There were significantly increased microglia activation and peripheral neutrophils infiltration at $24 \mathrm{~h}$ after SAH (Fig. 4). Immunofluorescence staining indicated that bexarotene treatment significantly decreased the number of activated Iba-1-positive microglia and the number of MPO-positive neutrophils in the basal cortex
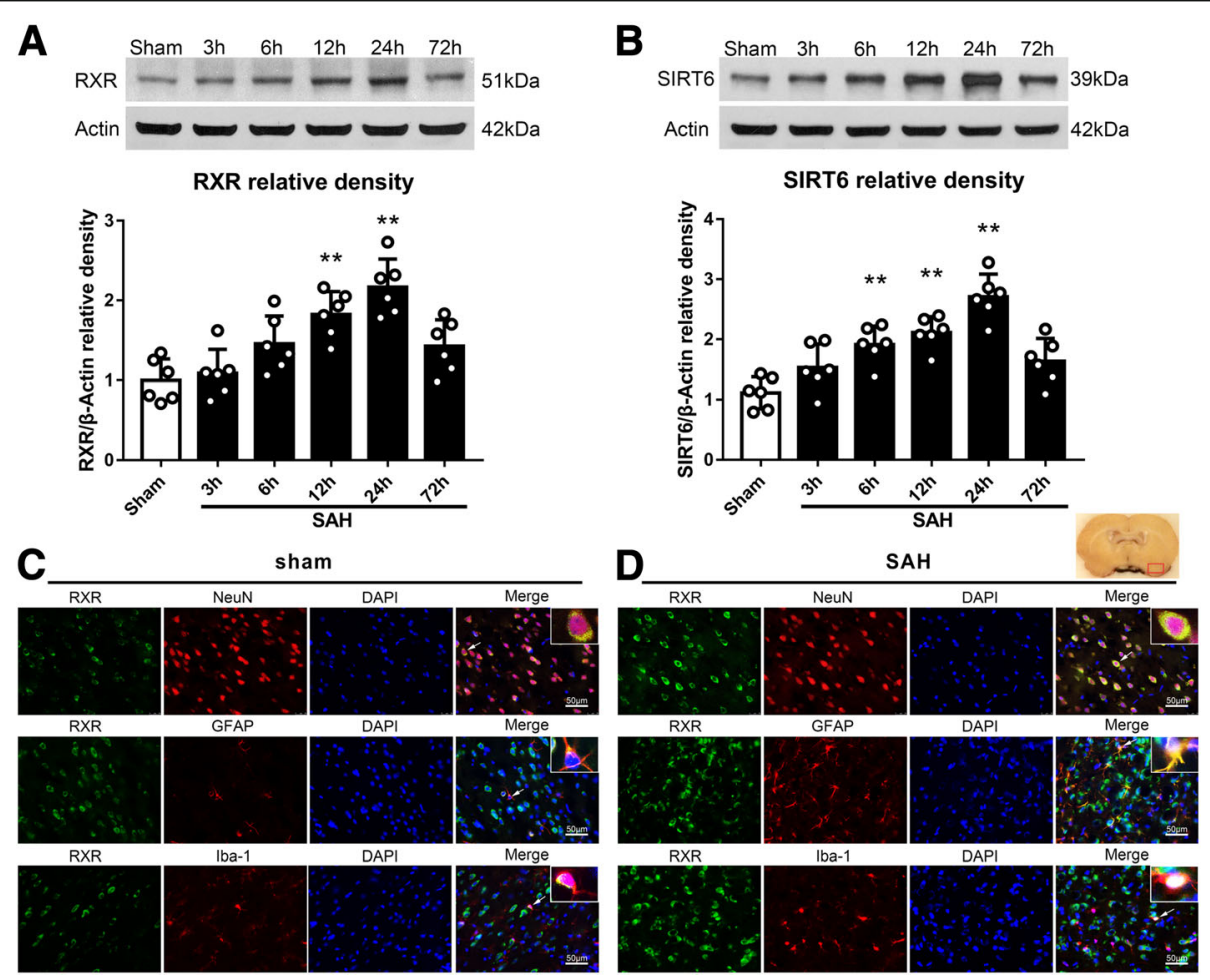

Fig. 2 Temporal expressions and cellular localization of endogenous brain RXR and SIRT6 after SAH. a Representative western blot bands and quantitative analyses of RXR time course in the left hemisphere after SAH. b Representative western blot bands and quantitative analyses of SIRT6 time course in the left hemisphere after SAH. ${ }^{*} P<0.05$ vs sham group. Error bars were represented as mean \pm SD. $n=6$ per group. $\mathbf{c}$ Double immunofluorescence staining for RXR (green) in the neuron (NeuN, red), astrocytes (GFAP, red), and microglia (Iba-1, red) in the left basal cortex at $24 \mathrm{~h}$ after SAH. d Double immunofluorescence staining for SIRT6 (red) in the neuron (NeuN, green), astrocytes (GFAP, green), and microglia (Iba-1, green) in the left basal cortex at $24 \mathrm{~h}$ after $\mathrm{SAH} . n=2$ per group. Scale bar, $50 \mu \mathrm{m}$. DAPI indicates 4',6-diamidino2phenylindole; GFAP glial fibrillary acidic protein, Iba-1 ionized calcium binding adaptor molecule-1, NeuN neuronal nuclear 

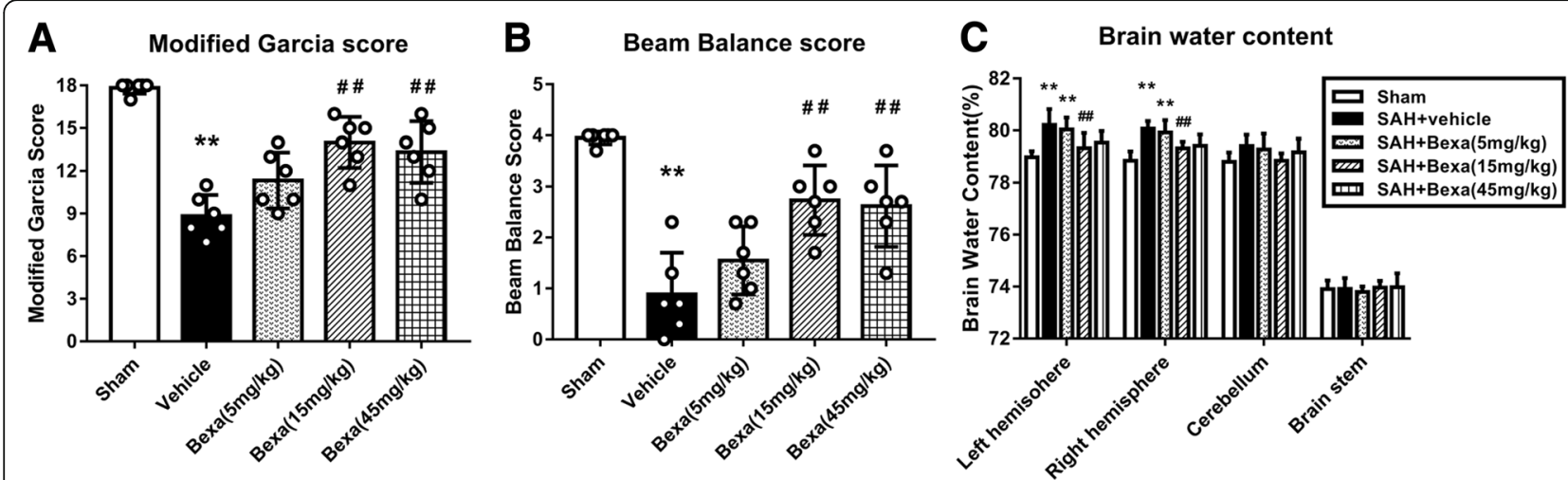

Fig. 3 Bexarotene attenuated the neurobehavioral deficits and brain edema at $24 \mathrm{~h}$ after SAH. a Modified Garcia score and beam balance score. b Brain water content in the left hemisphere, right hemisphere, cerebellum, and brain stem. Error bars were represented as mean $\pm S D$. $n=6$ per group. Bexa bexarotene

area of SAH rats $(P<0.05$, Fig. 4a, b, d, e). Consistently, the Western blot results showed that the protein levels of Iba-1 and MPO in the left hemisphere were significantly decreased by bexarotene treatment when compared with vehicle-treated SAH rats at $24 \mathrm{~h}$ after SAH $(P<0.05$, Fig. $4 \mathrm{c}, \mathrm{f})$.
Bexarotene increased the RXR/PPARY interaction at $24 \mathrm{~h}$ after SAH

Immunoprecipitation was applied to detect the RXR/ PPAR $\gamma$ interaction by bexarotene administration at $24 \mathrm{~h}$ after SAH. The IP result showed that the RXR interact with PPARy in sham and vehicle conditions; however, it

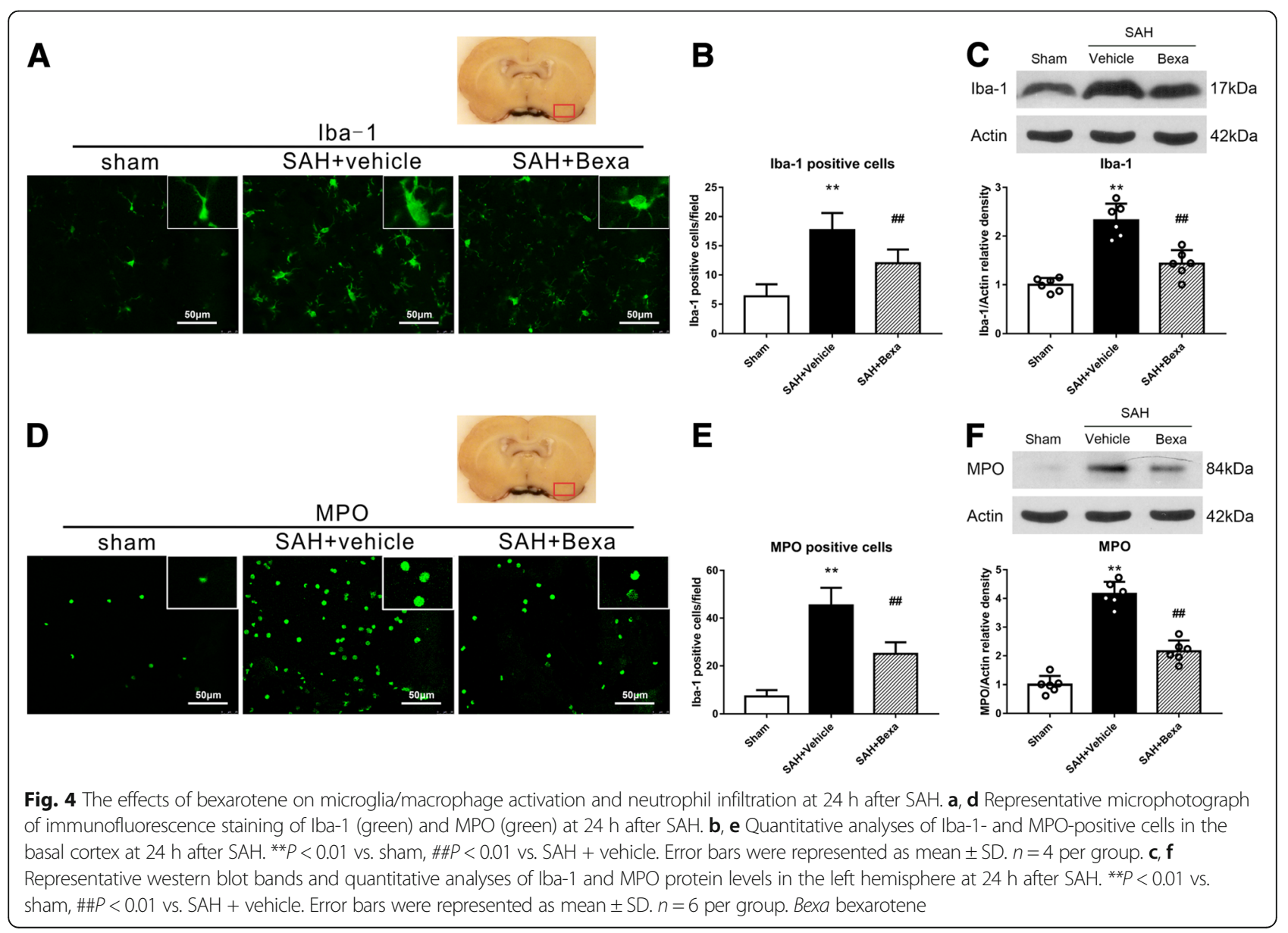




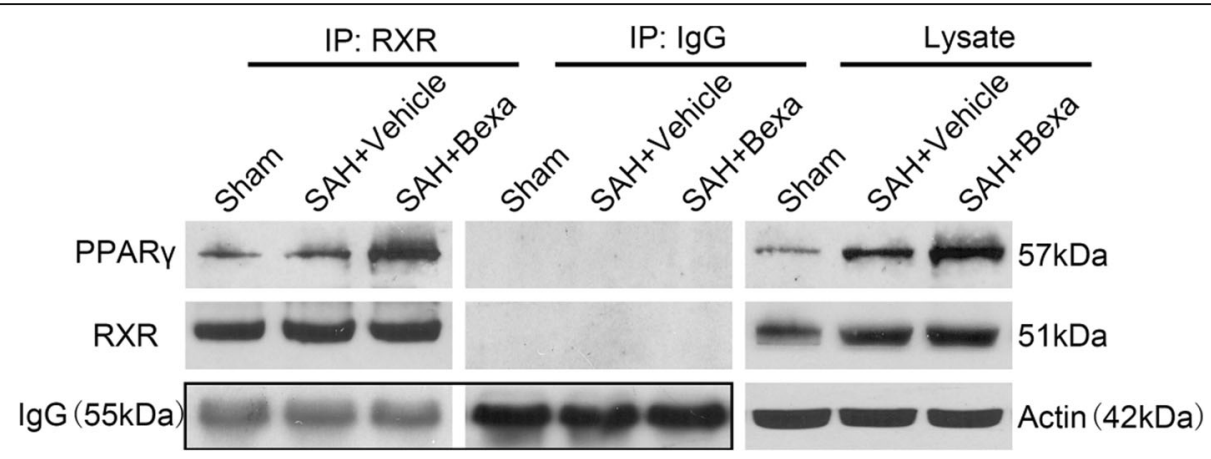

Fig. 5 The effects of bexarotene on the RXR/ PPARY interaction after SAH. IP assay of RXR/PPARY interaction in left hemisphere at $24 \mathrm{~h}$ after SAH. Bexa bexarotene

was increased after bexarotene treatment (Fig. 5). These results suggested that the formation of RXR/ PPAR $\gamma$ heterodimer were increased after bexarotene administration.

\section{Bexarotene treatment improved long-term neurobehavioral deficits after SAH}

Both the rotarod test and Morris water maze test were used to access the long-term neurological functions after SAH. In the rotarod test, the SAH + vehicle group had a significantly shorter falling latency at both $5 \mathrm{rpm}$ and $10 \mathrm{rpm}$ accelerating velocity tests when compared with the sham group in the 1 and 2 weeks after SAH. However, bexarotene treatment improved the rotarod performance of SAH rats at both $5 \mathrm{rpm}$ and $10 \mathrm{rpm}$ velocities compared to $\mathrm{SAH}+$ vehicle group $(P<0.05$; Fig. 6a).

In the Morris water maze, the escape latency and swim distance for the rats to find the platform were significantly increased in the $\mathrm{SAH}+$ vehicle group when compared to the sham group $(P<0.05$, Fig. $6 \mathrm{~b})$. However, a significant decrease in escape latency on days 3 to 4 and a significantly shorter swim distance on block 3 to 4 were observed in the SAH + Bexa group compared to the $\mathrm{SAH}+$ vehicle group $(P<$ 0.05 , Fig. $6 \mathrm{~b})$. In the probe quadrant trials, the percentage of time spent in the target quadrant was
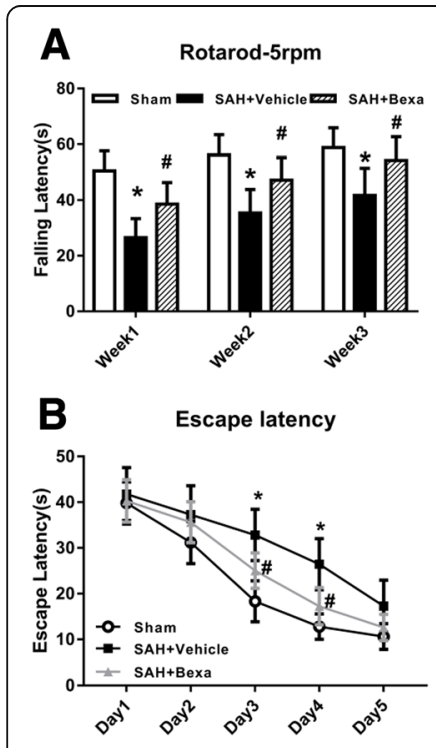
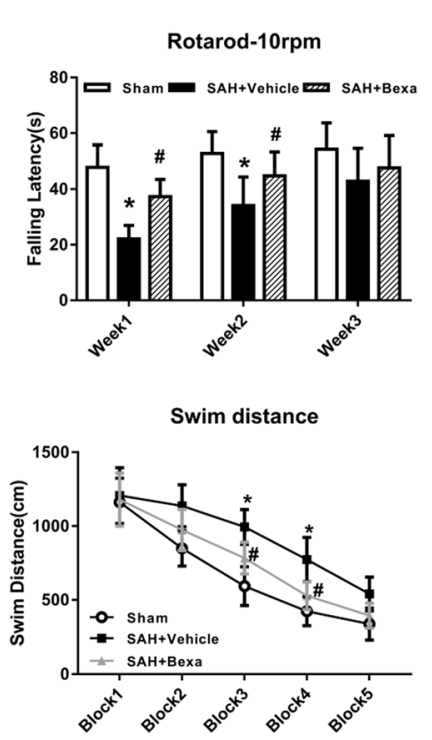
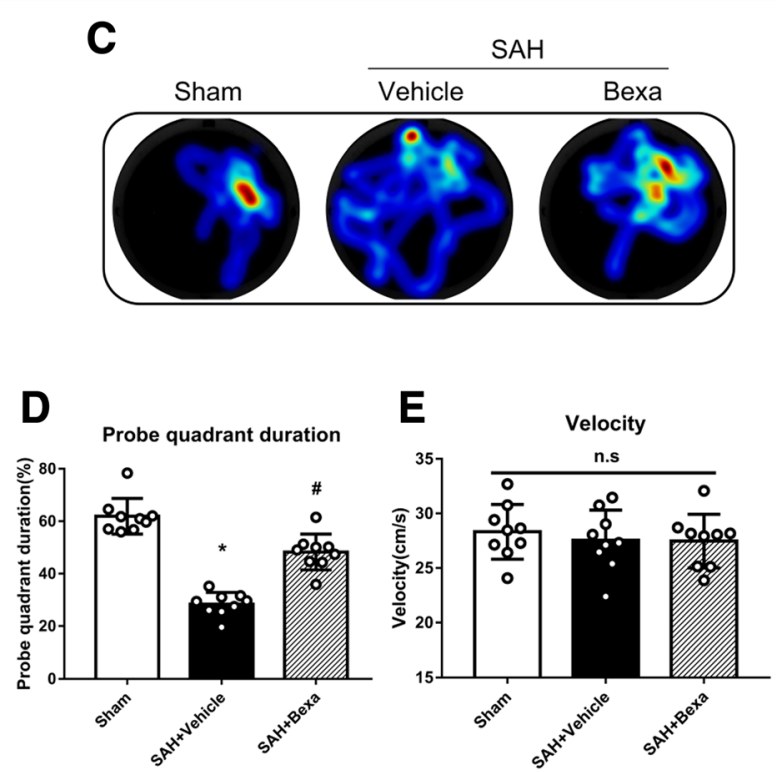

Fig. 6 Bexarotene improved the long-term neurological deficit after SAH. a Rotarod test of 5 rpm and 10 rpm. b Escape latency and swim distance of water maze test. c Representative heat map in probe test showed bexarotene treated SAH spent more time in probe quadrant. $\mathbf{d}$ Probe quadrant duration. e velocity of probe trial. ${ }^{*} P<0.05$, vs. sham group; $\# P<0.05$, vs. SAH + vehicle group. Data was represented as mean \pm SD. $n=9$ per group. Bexa bexarotene 
notably decreased in SAH + vehicle group compared with that in the sham group. However, bexarotene significantly increased the time spent in the target quadrant $(P<0.05$; Fig. 6c, d). The swimming speed was not significantly different among all the groups (Fig. 6e).

RXR antagonist UVI3003 reversed the antineuroinflammation effects of bexarotene and its regulation on PPARY/SIRT6/p-FoxO3a protein levels at $24 \mathrm{~h}$ after $\mathrm{SAH}$

To verify the RXR activation involved in the neuroprotective effects of bexarotene post-SAH, the specific RXR antagonist UVI3003 was injected via i.c.v to inhibit the RXR. Without changing RXR protein level, bexarotene further increased protein levels of PPAR $\gamma$ and SIRT6 but decreased p-FoxO3a and pro-inflammatory cytokines including IL-1 $\beta$, IL-6, and TNF-a in SAH rats when compared with those in the SAH + vehicle group $(P<0.05$; Fig. 7). UVI3003 reversed these effects of bexarotene after SAH $(P<0.05$; Fig. 7$)$.
RXR knockdown reversed the anti-neuroinflammation effects of bexarotene and its regulation on PPARY/SIRT6/ p-Fox03a protein levels at $24 \mathrm{~h}$ after SAH

To further verify if the RXR mediated the neuroprotective effects of bexarotene post-SAH, rat RXR siRNA was injected by i.c.v to knockdown RXR in vivo. The expression of RXR was significantly decreased by RXR siRNA compared to scramble siRNA. Also, without affected PPARY protein level, the downstream SIRT6 was decreased but increased p-FoxO3a and pro-inflammatory cytokines including IL-1 $\beta$, IL-6, and TNF-a in RXR knockdown group when compared with scramble siRNA group $(P<0.05$; Fig. 8$)$. The results suggested that knockdown RXR reversed the neuroprotective effect of bexarotene after SAH.

Selective SIRT6 inhibitor OSS128167 abolished the antineuroinflammation effects of bexarotene and its regulation on p-FoxO3a protein level at $24 \mathrm{~h}$ after SAH To verify if the SIRT6 was the downstream signaling of RXR activation by bexarotene, the selective SIRT6 inhibitor OSS128167 was injected via i.c.v to intervene
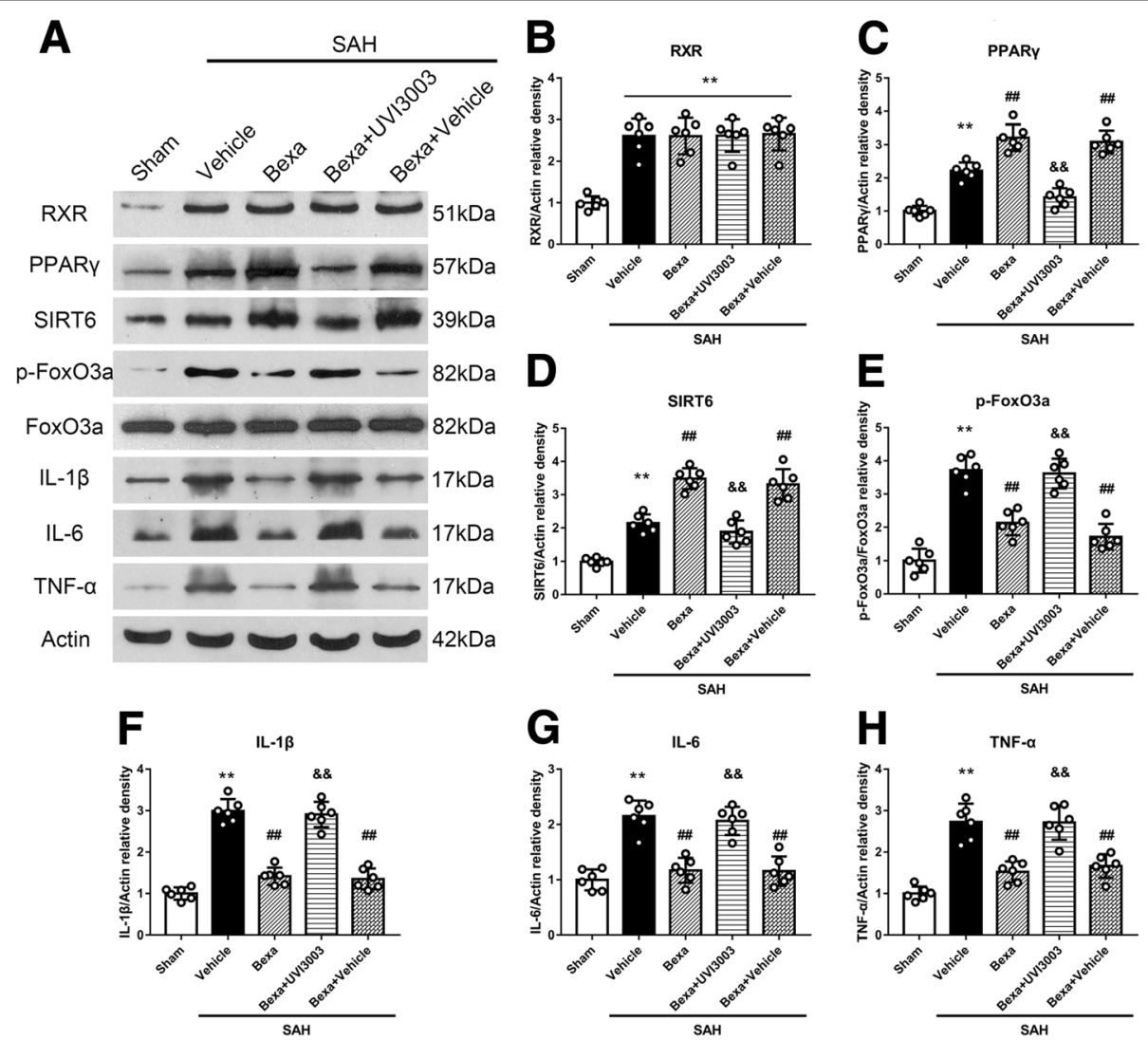

Fig. 7 RXR selective inhibitor UVI3003 reversed the anti-neuroinflammation effects of bexarotene and its regulation on downstream signaling proteins of PPARY/SIRT6/p-FoxO3a at $24 \mathrm{~h}$ after SAH. a Representative western blot bands. b-h Quantitative analyses of RXR, PPARY, SIRT6, p-FoxO3a, IL-1 3 , IL-6, and TNF-a in the left hemisphere at $24 \mathrm{~h}$ after SAH. ${ }^{* *} P<0.01$ vs. sham, $\# \# P<0.01 \mathrm{vs}$. SAH + vehicle, and $\& \& P<0.01$ vs. SAH + BEXA + vehicle. Error bars were represented as mean \pm SD. $n=6$ per group. Bexa bexarotene 


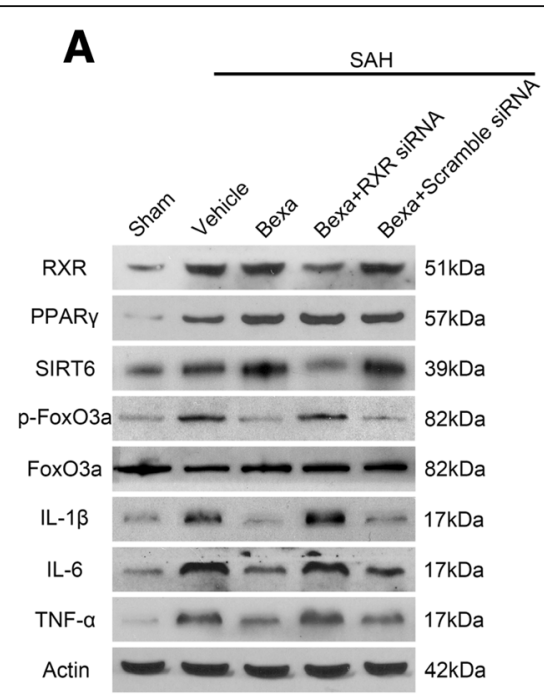

$\mathbf{F}$

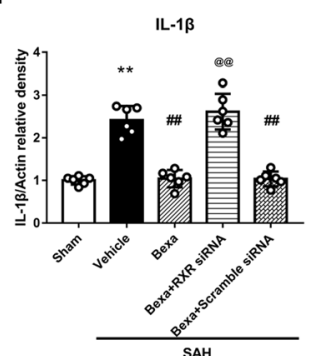

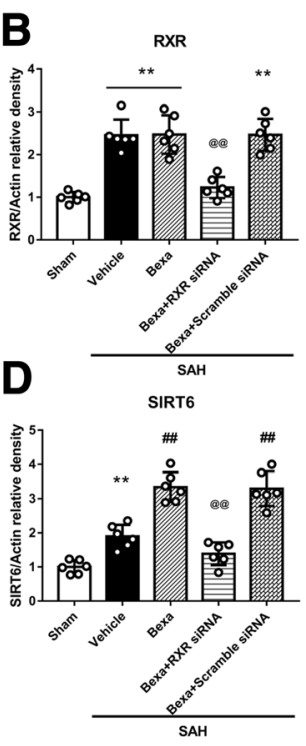

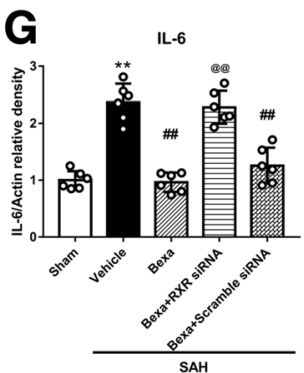

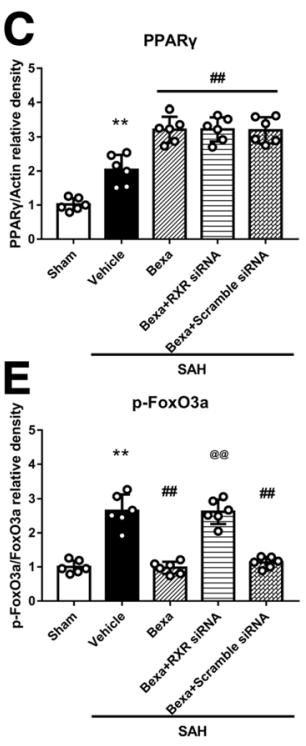

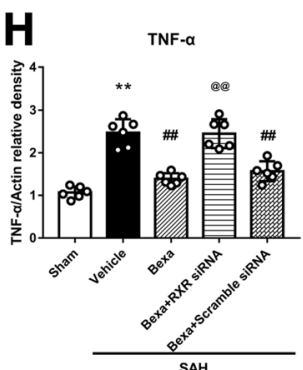

Fig. 8 RXR knockdown reversed the anti-neuroinflammation effects of bexarotene at $24 \mathrm{~h}$ after SAH. a Representative Western blot bands. b-h Quantitative analyses of RXR, PPARY, SIRT6, p-FoxO3a, IL-1 $\beta$, IL-6, and TNF- $a$ in the left hemisphere at $24 \mathrm{~h}$ after SAH. ${ }^{*} P<0.01$ vs. sham, \#\#P<0.01 vs. $\mathrm{SAH}+$ vehicle, and @@P $<0.01$ vs. SAH + BEXA + scramble siRNA. Error bars were represented as mean \pm SD. $n=6$ per group. Bexa bexarotene

the pathway of PPARy/SIRT6/FoxO3a. The change of the proteins levels in the signaling pathway after bexarotene treatment was the same as Figs. 7 and 8. However, inhibiting SIRT6 by OSS128167 abolished the effects of bexarotene on p-FoxO3a and pro-inflammatory cytokines IL-1 $\beta$, IL-6 and TNF-a $(P<0.05$; Fig. $8 \mathrm{a}, \mathrm{e}-\mathrm{h})$, but not the expression of PPARY and SIRT6 $(P>0.05$; Fig. $9 \mathrm{a}-\mathrm{C})$.

\section{Bexarotene attenuated SAH-induced IL-1 $\beta$-positive cells} and neutrophil infiltration which were abolished by either UVI3003 or OSS128167 at $24 \mathrm{~h}$ after SAH

Immunofluorescence staining of IL-1 $\beta$ and MPO were performed to confirm further that UVI3003 and OSS128167 abolished the anti-neuroinflammation effect of bexarotene after SAH. While the numbers of IL-1 $\beta$ - and MPO-positive cells were notably increased at $24 \mathrm{~h}$ after $\mathrm{SAH}$, bexarotene treatment significantly inhibited these pro-inflammatory responses. The administration of either UVI3003 or OSS128167 significantly reversed the anti-neuroinflammation effects of bexarotene after SAH $(P<0.05$, Fig. 10).

\section{Discussion}

In the present study, we explored the neuroprotective effects of bexarotene and the potential underlying mechanisms after experimentally induced SAH in rats. Our results demonstrated that (1) brain RXR protein level was increased at an early stage and peaked at $24 \mathrm{~h}$ after SAH. RXR expressions were co-localized with neurons, astrocytes, and microglia. (2) Activation RXR by bexarotene improved short- and long-term neurobehavioral outcomes, ameliorated brain edema, as well as inhibited the microglial activation and neutrophil infiltration after SAH. (3) Bexarotene treatment significantly increased expressions of PPAR $\gamma$ and SIRT6 but decreased the FoxO3a phosphorylation and the expressions of pro-inflammatory cytokines. (4) RXR antagonist UVI3002, RXR knockdown, or SIRT6 inhibitor OSS12817 abolished the anti-neuroinflammatory effects of bexarotene and its effects on PPARY/SIRT6/FoxO3a signaling pathway. Taken together, our results indicated that RXR activation with bexarotene might ameliorate neuroinflammation after SAH via at least partially the PPAR $\gamma /$ SIRT6/FoxO3a pathway. 

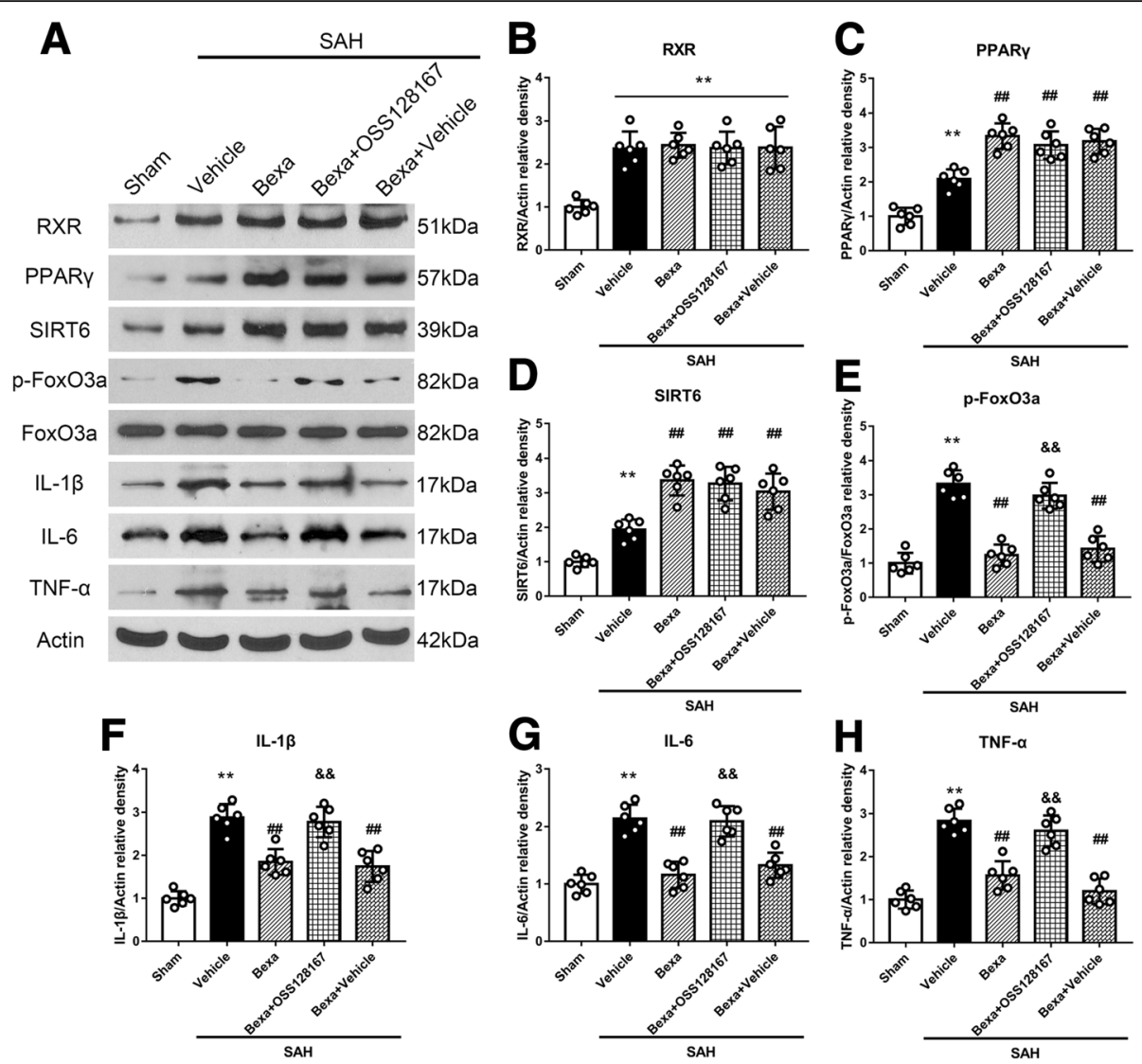

Fig. 9 SIRT6 inhibitor OSS128167 reversed the anti-neuroinflammation effect of bexarotene and its regulation on downstream signaling protein of p-FoxO3a at $24 \mathrm{~h}$ after SAH. a Representative Western blot bands. $\mathbf{b}-\mathbf{h}$ Quantitative analyses of RXR, PPARY, SIRT6, p-FoxO3a, IL-1 $\beta$, IL-6, and TNF-a in the left hemisphere at $24 \mathrm{~h}$ after SAH. ${ }^{*} P<0.01$ vs. sham, \#\#P<0.01 vs. SAH + vehicle, and $\& \& P<0.01$ vs. SAH + Bexa + vehicle. Error bars were represented as mean \pm SD. $n=6$ per group. Bexa bexarotene

Retinoid X receptor (RXR) is a nuclear factor widely expressed in the brain. Upon activation by binding to its ligands, RXR display a variety of biological functions [32]. Mounting of pieces of evidence suggested that RXR plays a vital role in the innate as well as in the adaptive immune response by regulating functions of monocytes/macrophages, dendritic cells, and T cells $[6,33]$. In the present study, our results showed that the expression of RXR increased at the early stage of SAH, and co-localized with neurons, astrocytes, and microglia. The increased RXR expression may suggest its participation in the endogenous neuroprotection mechanisms after SAH. Cells uptake retinol from the bloodstream and convert it into retinoic acid. The 9-cis retinoic acid (9cRA) is considered to be the endogenous ligand that activates RXR to manifest the subsequent transcriptional effects [34, 35]. However, the efficacy of endogenous 9cRA in RXR activation is limited since it is usually under detectable concentration in the brain after stroke [36].

Bexarotene is a highly selective and blood-brain barrier permeable agonist of RXR, Additional file 2: Figure S3), which is initially approved by FDA for treating cutaneous T cell lymphoma [37]. Emerging studies demonstrated that bexarotene was neuroprotective by reducing inflammation, attenuating apoptosis, enhancing autophagy, and promoting myelin debris phagocytosis in the central nervous system (CNS) diseases such as Alzheimer's disease, traumatic brain injury, and ischemic stroke $[7,16,19,38-41]$. Consistent with the previous studies, our results showed that bexarotene treatment improved neurological deficit, reduced the brain edema, and attenuated the microglial activation and neutrophil infiltration at $24 \mathrm{~h}$ after SAH. Because inflammation causes blood-brain barrier disruption which exacerbates brain edema after SAH $[8,42]$, this also can be explained as bexarotene can further improve neurological outcome by ameliorating brain edema exacerbated by inflammation. Additionally, the hippocampal damage at an early stage after SAH could cause long-term cognitive and memory impairment [43]. In the present study, bexarotene single administration improved long-term neurobehavioral functions including the performance of rotarod 


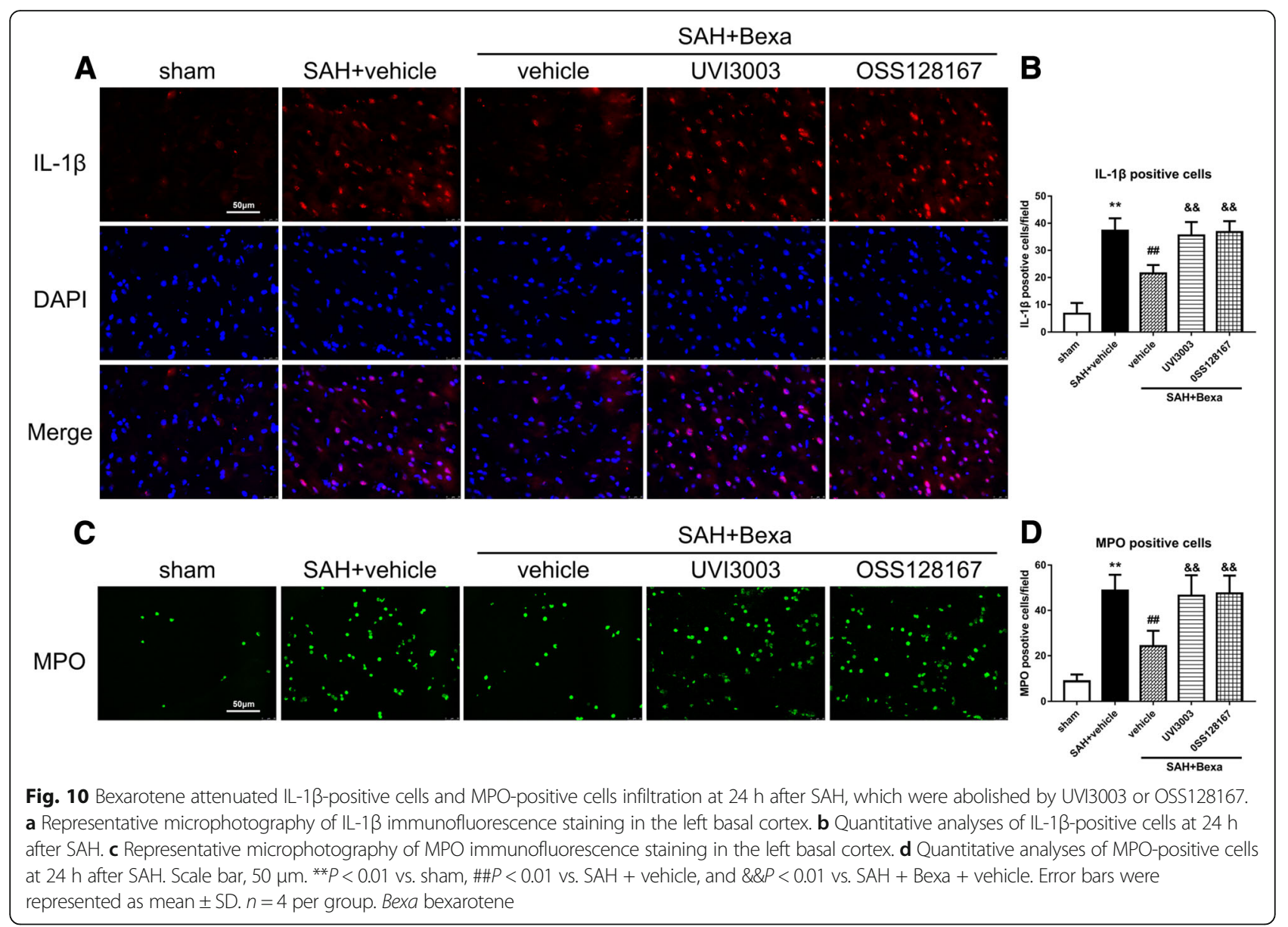

test and Morris water maze spatial learning test. The long-term protection may be attributed to the attenuated EBI by RXR activation after SAH.

Heterodimers of RXR with PPARs are the most extensively studied nuclear receptors involved in immune responses that play crucial roles in apoptotic cell clearance, neutrophil homeostasis, immune cell proliferation, $\mathrm{T}$ cell differentiation, and inflammatory gene repression [44]. The $\mathrm{RXR} / \mathrm{PPAR} \gamma$ heterodimer activation has been shown to be neuroprotective in CNS diseases [7, 45, 46]. Recent studies indicated that the PPAR $\gamma$ activation showed a protective effect by increasing SIRT6 expression in animal models of hepatic steatosis and Huntington's disease (HD) [13, 14]. Consistently, in the present study, our results demonstrated that administration of bexarotene significantly increased the RXR/PPAR $\gamma$ interaction, and upregulated the expression of SIRT6, while downregulating the pro-inflammatory cytokines including IL-1 $\beta$, IL-6, and TNF- $\alpha$ at $24 \mathrm{~h}$ after SAH. UVI3003, a selective RXR antagonist [32, 47, 48], could ultimately prevent the beneficial effects of RXR activation in retinal degenerations [49]. Similarly, when we inhibited RXR using UVI3003 or knockdown RXR before SAH induction, the effects of bexarotene treatment on PPAR $\gamma /$ SIRT6 upregulation and neuroinflammation suppression as well as neurological deficit improvement were abolished at $24 \mathrm{~h}$ after $\mathrm{SAH}$.

Both in vitro and in vivo studies demonstrated that SIRT6 produced beneficial effects in cerebrovascular diseases models [50-52]. SIRT6 was essential for the sodium sulfide-mediated cytoprotective effect in brain endothelial cells subjected to ischemia/reperfusion (I/R) injury [51]. SIRT6 protected the brain from cerebral I/R injury [50] and contributed to neurogenesis after cerebral ischemia [52]. Moreover, SIRT6 protected cardiomyocytes against I/R injury by a FoxO3a-dependent mechanism, in which SIRT6 decreased cellular oxidative stress by inhibiting FoxO3a phosphorylation and subsequently upregulating the antioxidant-encoding gene expression [15]. Consistently, we found that bexarotene treatment significantly increased SIRT6 expression but decrease the expression of p-FoxO3a and pro-inflammatory cytokines. The application of OSS128167, a selective SIRT6 inhibitor, reversed the neurological improvement and anti-inflammatory effects of bexarotene and its down-regulation on p-FoxO3a expression after SAH. Mechanistically, the RXR inhibitor, RXR knockdown, and 
SIRT6 inhibitor intervene the pathway, reversed the neuroscore improvement (Additional file 3: Figure S2) and the anti-neuroinflammatory effect marked by pro-inflammatory cytokines after SAH treated by bexarotene. Taken together, our results suggested that PPAR $\gamma /$ SIRT6/FoxO3a signaling pathway may underline the anti-neuroinflammation and neurological improvement of bexarotene mediated by RXR after $\mathrm{SAH}$.

There were some limitations to the present study. First, we focused on the anti-inflammatory effects of bexarotene after $\mathrm{SAH}$, but we cannot exclude the possibility that bexarotene may also exert other protective effects, such as preservation of BBB integrity, modulation of autophagy, and regulation neurogenesis $[16,39,53]$. Second, bexarotene administration was only applied at a single time point (1 $\mathrm{h}$ after SAH). Thus the optimal therapeutic window of bexarotene treatment for SAH was not evaluated in the present study. Third, a previous study reported that activation of PPAR $\gamma$ attenuated NF- $\mathrm{kB}$-mediated inflammation [8]. Therefore, there may be other downstream signaling pathways underlying the anti-inflammation effects of bexarotene. Future studies are required to fully elucidate the other neuroprotective functions and potential mechanisms associated with bexarotene in the setting of experimental SAH.

\section{Conclusions}

Our findings demonstrated that the activation of RXR with bexarotene improved the short- and long-term neurological functions, and attenuated neuroinflammation after $\mathrm{SAH}$ in rats. The protective effects of bexarotene were at least in part through PPAR $/$ /SIRT6/FoxO3a signaling pathway. Therefore, bexarotene may provide a promising therapeutic strategy for the management of SAH patients.

\section{Additional files}

Additional file 1: Figure S1. A. Numbers of animals used, mortality and exclusion in each group. B. Representative brain images in sham and $\mathrm{SAH}$ groups. Blood clots were mainly present around the Circle of Willis at $24 \mathrm{~h}$ after SAH. C. SAH grade scores of all SAH groups at $24 \mathrm{~h}$ after SAH. Bexa, bexarotene; UVI3003, a specific RXR antagonist; OSS128167, a selective SIRT6 inhibitor; Scr siRNA, Scramble siRNA; SAH, subarachnoid hemorrhage. (TIF $7668 \mathrm{~kb}$ )

Additional file 2: Figure S3. Detection of bexarotene in the brain after intraperitoneal administration. A. Mass spectra of bexarotene detected with full scan mass spectrometry (MS). B. MS/MS spectra of precursor ion at $\mathrm{m} / \mathrm{z} 349$ from bexarotene standard. C. MS/MS spectra of precursor ion at $\mathrm{m} / \mathrm{z} 349$ from the brain of dosed rats. (TIF $3101 \mathrm{~kb}$ )

Additional file 3: Figure S2. Bexarotene improves neurological deficit at $24 \mathrm{~h}$ after SAH, however the neuroprotective effect can be reversed by UVI3003, RXR knockdown and OSS128167 administration. A. Modified Garcia score. B. Beam balance test. ${ }^{*} p<0.01$ vs. sham, $\# \# p<0.01$ vs. SAH + vehicle, and $\& \& p<0.01$ vs. SAH + Bexa + vehicle. $@ @ p<0.01$ vs. SAH + Bexa + Scr siRNA. Error bars were represented as mean \pm SD. $n=6$ per group. Bexa, bexarotene; Scr siRNA, scramble siRNA. (TIF $1731 \mathrm{~kb}$ )

\section{Abbreviations}

9cRA: 9-cis retinoic acid; AD: Alzheimer's disease; ANOVA: Analysis of variance; Bexa: Bexarotene; CNS: Central nervous system; DMSO: Dimethyl sulfoxide; EBI: Early brain injury; FDA: Food and Drug Administration; FoxO3a: Forkhead box O3a; GFAP: Glial fibrillary acidic protein; HD: Huntington's disease; i.c.v: Intracerebroventricular; i.p: Intraperitoneal; I/ R: Ischemia/reperfusion; Iba-1: Ionized calcium binding adaptor molecule 1 ; MPO: Myeloperoxidase; NeuN: Neuronal nuclear; p-FoxO3a: Phosphate forkhead box O3a; PPARY: Peroxisome proliferator-activated receptor gamma; RXR: Retinoid X receptor; SAH: Subarachnoid hemorrhage; SD: Standard deviation; SIRT6: Sirtuin 6

\section{Acknowledgements}

Not applicable.

\section{Funding}

This study was supported by grants from the National Institutes of Health NS081740 and NS 082184 to Dr. J.H. Zhang and Grant 81571150 and 81870944 from the National Natural Science Foundation of China to Dr. F. Liu.

Availability of data and materials

All data generated or analyzed during this study are included in this published article.

\section{Authors' contributions}

Y.Z., B.E., J.T., F.L., and J.Z. participated in the experimental design, data analysis and interpretation, and manuscript preparation. Y.Z., W.X., O.U., and G.Z. performed the experiments. Y.Z. and W.X. collected and analyzed the data. Y.Z. and L.H. drafted the manuscript and Z.T. proofread the language. All authors read and approved the final manuscript.

\section{Ethics approval and consent to participate}

All animal experiments in this study have been approved by the Institutional Animal Care and Use Committee of Loma Linda University in accordance with the National Institutes of Health (NIH) Guide for the Care and Use of Laboratory Animals.

Consent for publication

Not applicable.

Competing interests

The authors declare that they have no competing interests.

\section{Publisher's Note}

Springer Nature remains neutral with regard to jurisdictional claims in published maps and institutional affiliations.

\section{Author details}

'Department of Neurosurgery, Third XiangYa Hospital, Central South University, No.138 Tongzipo Road, Changsha 410013, China. ${ }^{2}$ Department of Physiology and Pharmacology, Loma Linda University, 11041 Campus St. Risley Hall, Loma Linda, CA 92354, USA. ${ }^{3}$ Department of Neurosurgery, School of Medicine, Loma Linda University, Loma Linda, CA 92354, USA. ${ }^{4}$ Department of Anesthesiology, School of Medicine, Loma Linda University, Loma Linda, CA 92354, USA. ${ }^{5}$ Mass Spectrometry Core Facility, Loma Linda University, Loma Linda, CA 92354, USA. ${ }^{6}$ Department of Earth and Biological Sciences, School of Medicine, Loma Linda University, Loma Linda, CA 92354, USA.

Received: 19 December 2018 Accepted: 4 February 2019

Published online: 21 February 2019

\section{References}

1. van Gijn J, Kerr RS, Rinkel GJ. Subarachnoid haemorrhage. Lancet. 2007;369: 306-18.

2. Lapchak PA, Zhang JH. The high cost of stroke and stroke cytoprotection research. Transl Stroke Res. 2017:8:307-17.

3. Suzuki H. What is early brain injury? Transl Stroke Res. 2015;6:1-3.

4. Aragon MJ, Topper L, Tyler CR, Sanchez B, Zychowski K, Young T, Herbert G, Hall P, Erdely A, Eye T, et al. Serum-borne bioactivity caused by pulmonary 
multiwalled carbon nanotubes induces neuroinflammation via blood-brain barrier impairment. Proc Natl Acad Sci U S A. 2017;114:E1968-76.

5. Altucci L, Leibowitz MD, Ogilvie KM, de Lera AR, Gronemeyer H. RAR and RXR modulation in cancer and metabolic disease. Nat Rev Drug Discov. 2007;6:793-810.

6. Roszer T, Menendez-Gutierrez MP, Cedenilla M, Ricote M. Retinoid X receptors in macrophage biology. Trends Endocrinol Metab. 2013;24:460-8.

7. Certo M, Endo Y, Ohta K, Sakurada S, Bagetta G, Amantea D. Activation of RXR/PPARgamma underlies neuroprotection by bexarotene in ischemic stroke. Pharmacol Res. 2015;102:298-307.

8. Xie Z, Huang L, Enkhjargal B, Reis C, Wan W, Tang J, Cheng Y, Zhang $J H$. Recombinant Netrin-1 binding UNC5B receptor attenuates neuroinflammation and brain injury via PPARgamma/NFkappaB signaling pathway after subarachnoid hemorrhage in rats. Brain Behav Immun. 2018:69:190-202.

9. Gu C, Wang Y, Li J, Chen J, Yan F, Wu C, Chen G. Rosiglitazone attenuates early brain injury after experimental subarachnoid hemorrhage in rats. Brain Res. 2015;1624:199-207.

10. Drew PD, Johnson JW, Douglas JC, Phelan KD, Kane CJ. Pioglitazone blocks ethanol induction of microglial activation and immune responses in the hippocampus, cerebellum, and cerebral cortex in a mouse model of fetal alcohol spectrum disorders. Alcohol Clin Exp Res. 2015;39:445-54.

11. Collino M, Patel NS, Thiemermann C. PPARs as new therapeutic targets for the treatment of cerebral ischemia/reperfusion injury. Ther Adv Cardiovasc Dis. 2008;2:179-97.

12. Zhao XR, Gonzales N, Aronowski J. Pleiotropic role of PPARgamma in intracerebral hemorrhage: an intricate system involving Nrf2, RXR, and NF-kappaB. CNS Neurosci Ther. 2015;21:357-66.

13. Yang SJ, Choi JM, Chae SW, Kim WJ, Park SE, Rhee EJ, Lee WY, Oh KW, Park SW, Kim SW, Park CY. Activation of peroxisome proliferatoractivated receptor gamma by rosiglitazone increases sirt6 expression and ameliorates hepatic steatosis in rats. PLoS One. 2011:6:e17057.

14. Jin J, Albertz J, Guo Z, Peng Q, Rudow G, Troncoso JC, Ross CA, Duan $W$. Neuroprotective effects of PPAR-gamma agonist rosiglitazone in N171-82Q mouse model of Huntington's disease. J Neurochem. 2013; 125:410-9.

15. Wang XX, Wang XL, Tong MM, Gan L, Chen H, Wu SS, Chen JX, Li RL, Wu Y, Zhang HY, et al. SIRT6 protects cardiomyocytes against ischemia/reperfusion injury by augmenting FoxO3alpha-dependent antioxidant defense mechanisms. Basic Res Cardiol. 2016;111:13.

16. Zhong J, Cheng C, Liu H, Huang Z, Wu Y, Teng Z, He J, Zhang H, Wu J, Cao $F$, et al. Bexarotene protects against traumatic brain injury in mice partially through apolipoprotein E. Neuroscience. 2017;343:434-48.

17. Riancho J, Berciano MT, Berciano J, Lafarga M. Relaunching an old drug: the potential role of bexarotene in neurodegenerative diseases. J Neurol. 2016; 263:177-8.

18. Dheer Y, Chitranshi N, Gupta V, Abbasi M, Mirzaei M, You Y, Chung R, Graham SL, Gupta V. Bexarotene modulates retinoid-X-receptor expression and is protective against neurotoxic endoplasmic reticulum stress response and apoptotic pathway activation. Mol Neurobiol. 2018;55:9043-56.

19. Cramer PE, Cirrito JR, Wesson DW, Lee CY, Karlo JC, Zinn AE, Casali BT, Restivo $\mathrm{J}$, Goebel WD, James MJ, et al. ApoE-directed therapeutics rapidly clear beta-amyloid and reverse deficits in AD mouse models. Science. 2012; 335:1503-6.

20. Siler DA, Gonzalez JA, Wang RK, Cetas JS, Alkayed NJ. Intracisterna administration of tissue plasminogen activator improves cerebrospinal fluid flow and cortical perfusion after subarachnoid hemorrhage in mice. Transl Stroke Res. 2014;5:227-37.

21. Muroi C, Fujioka M, Mishima K, Irie K, Fujimura Y, Nakano T, Fandino J, Keller E, Iwasaki K, Fujiwara M. Effect of ADAMTS-13 on cerebrovascular microthrombosis and neuronal injury after experimental subarachnoid hemorrhage. J Thromb Haemost. 2014;12:505-14.

22. Iniaghe LO, Krafft PR, Klebe DW, Omogbai EKI, Zhang JH, Tang J. Dimethyl fumarate confers neuroprotection by casein kinase 2 phosphorylation of Nrf2 in murine intracerebral hemorrhage. Neurobiol Dis. 2015;82:349-58.

23. Chen S, Zhao L, Sherchan P, Ding Y, Yu J, Nowrangi D, Tang J, Xia Y, Zhang $\mathrm{JH}$. Activation of melanocortin receptor 4 with RO27-3225 attenuates neuroinflammation through AMPK/JNK/p38 MAPK pathway after intracerebral hemorrhage in mice. J Neuroinflammation. 2018;15:106.

24. Altay O, Suzuki H, Hasegawa Y, Ostrowski RP, Tang J, Zhang JH. Isoflurane on brain inflammation. Neurobiol Dis. 2014;62:365-71.
25. Sugawara $T$, Ayer R, Jadhav V, Zhang JH. A new grading system evaluating bleeding scale in filament perforation subarachnoid hemorrhage rat model. J Neurosci Methods. 2008;167:327-34.

26. Liu L, Fujimoto M, Nakano F, Nishikawa H, Okada T, Kawakita F, ImanakaYoshida K, Yoshida T, Suzuki H. Deficiency of tenascin-C alleviates neuronal apoptosis and Neuroinflammation after experimental subarachnoid hemorrhage in mice. Mol Neurobiol. 2018;55:8346-54.

27. Xie Z, Enkhjargal B, Wu L, Zhou K, Sun C, Hu X, Gospodarev V, Tang J, You C, Zhang JH. Exendin-4 attenuates neuronal death via GLP-1R/PI3K/Akt pathway in early brain injury after subarachnoid hemorrhage in rats. Neuropharmacology. 2018;128:142-51.

28. Liu F, Chen Y, Hu Q, Li B, Tang J, He Y, Guo Z, Feng H, Tang J, Zhang $J$ H. MFGE8/integrin beta3 pathway alleviates apoptosis and inflammation in early brain injury after subarachnoid hemorrhage in rats. Exp Neurol. 2015;272:120-7.

29. Mo J, Enkhjargal B, Travis ZD, Zhou K, Wu P, Zhang G, Zhu Q, Zhang T, Peng J, Xu W, et al. AVE 0991 attenuates oxidative stress and neuronal apoptosis via Mas/PKA/CREB/UCP-2 pathway after subarachnoid hemorrhage in rats. Redox Biol. 2018;20:75-86.

30. Jiang Y, Wang J, Rozewski DM, Kolli S, Wu CH, Chen CS, Yang X, Hofmeister CC, Byrd JC, Johnson AJ, Phelps MA. Sensitive liquid chromatography/mass spectrometry methods for quantification of pomalidomide in mouse plasma and brain tissue. J Pharm Biomed Anal. 2014;88:262-8.

31. Xiao Y, Li G, Chen Y, Zuo Y, Rashid K, He T, Feng H, Zhang JH, Liu F. Milk fat globule-epidermal growth Factor-8 pretreatment attenuates apoptosis and inflammation via the integrin-beta3 pathway after surgical brain injury in rats. Front Neurol. 2018;9:96.

32. Hiebl V, Ladurner A, Latkolik S, Dirsch VM. Natural products as modulators of the nuclear receptors and metabolic sensors LXR, FXR and RXR. Biotechnol Adv. 2018:36:1657-98.

33. Pino-Lagos K, Guo Y, Noelle RJ. Retinoic acid: a key player in immunity. Biofactors. 2010;36:430-6.

34. Maden M. Retinoid signalling in the development of the central nervous system. Nat Rev Neurosci. 2002;3:843-53.

35. Yu SJ, Airavaara M, Wu KJ, Harvey BK, Liu HS, Yang Y, Zacharek A, Chen J, Wang $Y$. 9-cis retinoic acid induces neurorepair in stroke brain. Sci Rep. 2017;7:4512

36. Shen $H$, Luo $Y$, Kuo CC, Deng $X$, Chang CF, Harvey BK, Hoffer BJ, Wang $Y$. 9-Cis-retinoic acid reduces ischemic brain injury in rodents via bone morphogenetic protein. J Neurosci Res. 2009;87:545-55.

37. Jawed Sl, Myskowski PL, Horwitz S, Moskowitz A, Querfeld C. Primary cutaneous T-cell lymphoma (mycosis fungoides and Sezary syndrome): part II. Prognosis, management, and future directions. J Am Acad Dermatol. 2014;70(223):e221-17. quiz 240-222

38. Casali BT, Reed-Geaghan EG, Landreth GE. Nuclear receptor agonistdriven modification of inflammation and amyloid pathology enhances and sustains cognitive improvements in a mouse model of Alzheimer's disease. J Neuroinflammation. 2018;15:43.

39. Huuskonen MT, Loppi S, Dhungana H, Keksa-Goldsteine V, Lemarchant S, Korhonen P, Wojciechowski S, Pollari E, Valonen P, Koponen J, et al. Bexarotene targets autophagy and is protective against thromboembolic stroke in aged mice with tauopathy. Sci Rep. 2016;6:33176.

40. Amantea D, Bagetta G. Drug repurposing for immune modulation in acute ischemic stroke. Curr Opin Pharmacol. 2016;26:124-30.

41. Natrajan MS, de la Fuente AG, Crawford AH, Linehan E, Nunez $V$, Johnson KR, Wu T, Fitzgerald DC, Ricote M, Bielekova B, Franklin RJ. Retinoid X receptor activation reverses age-related deficiencies in myelin debris phagocytosis and remyelination. Brain. 2015;138:3581-97.

42. Zhu Q, Enkhjargal B, Huang L, Zhang T, Sun C, Xie Z, Wu P, Mo J, Tang J, Xie Z, Zhang JH. Aggf1 attenuates neuroinflammation and BBB disruption via PI3K/Akt/NF-kappaB pathway after subarachnoid hemorrhage in rats. Neuroinflammation. 2018;15:178.

43. Veldeman M, Coburn M, Rossaint R, Clusmann H, Nolte K, Kremer B, Hollig A. Xenon reduces neuronal hippocampal damage and alters the pattern of microglial activation after experimental subarachnoid hemorrhage: a randomized controlled animal trial. Front Neurol. 2017:8:511.

44. Huang W, Glass CK. Nuclear receptors and inflammation control: molecular mechanisms and pathophysiological relevance. Arterioscler Thromb Vasc Biol. 2010;30:1542-9.

45. Tu L, Yang XL, Zhang Q, Wang Q, Tian T, Liu D, Qu X, Tian JY. Bexarotene attenuates early brain injury via inhibiting micoglia activation through 
PPARgamma after experimental subarachnoid hemorrhage. Neurol Res. 2018;40:702-8.

46. Bonet-Costa V, Herranz-Perez V, Blanco-Gandia M, Mas-Bargues C, Ingles M, Garcia-Tarraga P, Rodriguez-Arias M, Minarro J, Borras C, Garcia-Verdugo JM, Vina J. Clearing amyloid-beta through PPARgamma/ApoE activation by Genistein is a treatment of experimental Alzheimer's disease. J Alzheimers Dis. 2016;51:701-11.

47. Cambray S, Arber C, Little G, Dougalis AG, de Paola V, Ungless MA, Li M, Rodriguez TA. Activin induces cortical interneuron identity and differentiation in embryonic stem cell-derived telencephalic neural precursors. Nat Commun. 2012;3:841.

48. Hebert SL, Fitzpatrick KR, McConnell SA, Cucak A, Yuan C, McLoon LK. Effects of retinoic acid signaling on extraocular muscle myogenic precursor cells in vitro. Exp Cell Res. 2017;361:101-11.

49. Lin Y, Jones BW, Liu A, Tucker JF, Rapp K, Luo L, Baehr W, Bernstein PS, Watt $\mathrm{CB}$, Yang $\mathrm{JH}$, et al. Retinoid receptors trigger neuritogenesis in retinal degenerations. FASEB J. 2012;26:81-92.

50. Zhang W, Wei R, Zhang L, Tan Y, Qian C. Sirtuin 6 protects the brain from cerebral ischemia/reperfusion injury through NRF2 activation. Neuroscience. 2017;366:95-104

51. Hu Y, Li R, Yang H, Luo H, Chen Z. Sirtuin 6 is essential for sodium sulfidemediated cytoprotective effect in ischemia/reperfusion-stimulated brain endothelial cells. J Stroke Cerebrovasc Dis. 2015;24:601-9.

52. Zhao Y, Guan YF, Zhou XM, Li GQ, Li ZY, Zhou CC, Wang P, Miao CY. Regenerative neurogenesis after ischemic stroke promoted by nicotinamide Phosphoribosyltransferase-nicotinamide adenine dinucleotide Cascade. Stroke. 2015;46:1966-74.

53. Mounier A, Georgiev D, Nam KN, Fitz NF, Castranio EL, Wolfe CM, Cronican AA, Schug J, Lefterov I, Koldamova R. Bexarotene-activated retinoid X receptors regulate neuronal differentiation and dendritic complexity. J Neurosci. 2015;35:11862-76.

Ready to submit your research? Choose BMC and benefit from:

- fast, convenient online submission

- thorough peer review by experienced researchers in your field

- rapid publication on acceptance

- support for research data, including large and complex data types

- gold Open Access which fosters wider collaboration and increased citations

- maximum visibility for your research: over $100 \mathrm{M}$ website views per year

At $\mathrm{BMC}$, research is always in progress.

Learn more biomedcentral.com/submissions 